\title{
FORMULATION AND EVALUATION OF TELMISARTAN SOLID DISPERSIONS USING ENTADA SCANDENS SEED STARCH AND POLOXAMER-188 AS SUPERDISINTEGRANTS
}

\author{
VENKATARAO MANNEM, VIDYADHARA SURYADEVARA*, SANDEEP DOPPALAPUDI
}

Department of Pharmaceutics, Chebrolu Hanumaiah Institute of Pharmaceutical Sciences, Chandramoulipuram, Chowdavaram, Guntur - 522 019, Andhra Pradesh, India. Email: svidyadhara@gmail.com

Received: 12 July 2018, Revised and Accepted: 06 August 2018

\section{ABSTRACT}

Objective: The current research focuses on solubility enhancement of poorly water-soluble drug telmisartan, using novel superdisintegrants such as Entada scandens seed starch and Poloxamer-188. Starches yielded from plants are pharmaceutically useful as binders, diluents, disintegrants, and lubricants.

Methods: Starches were extracted from E. scandens seed powder using alkali method (sodium hydroxide at $0.1 \%, 0.25 \%$, and $0.5 \%$ concentrations) and water. These starches were subjected for the evaluation of various physicochemical properties and phytochemical tests.

Results: The phytochemical tests revealed the presence of only starch in all the extracts. Of all the starches, the starch prepared from $0.5 \%$ sodium hydroxide (ESS4) showed best physicochemical properties. Solid dispersions were prepared using telmisartan, poloxamer-188, and starch (ESS4) in various concentrations using fusion technique. Various pre-formulation parameters were evaluated. From in vitro dissolution studies, it was observed that the solid dispersion formulation TP7 containing telmisartan and poloxamer-188 in 1:4 ratios showed better dissolution rate. Solid dispersion TPS7 containing TP7 formulation and $15 \% \mathrm{w} / \mathrm{w}$ of alkali extracted starch showed faster disintegration and enhanced dissolution rate than the solid dispersions prepared alone with poloxamer-188. Fourier transform infrared spectroscopy and differential scanning calorimetric studies for optimized formulations revealed that there were no major interactions between the drug and excipients. X-ray diffraction studies revealed the crystalline and amorphous nature of formulations.

Conclusion: Thus, the solid dispersions prepared using E. scandens seed starch revealed the superdisintegrant property of starch.

Keywords: Entada scandens, Solid dispersions, Telmisartan, Poloxamer-188.

(C) 2018 The Authors. Published by Innovare Academic Sciences Pvt Ltd. This is an open access article under the CC BY license (http://creativecommons. org/licenses/by/4. 0/) DOI: http://dx.doi.org/10.22159/ajpcr.2018.v11i9.28422

\section{INTRODUCTION}

Oral palatability of a dosage form is comfortable and familiar means of taking medication, according to the patient [1]. Although it is much preferred, limited drug absorption which results in poor bioavailability is a major issue that can be observed while delivering an active agent through oral route. This may be attributed to various factors such as poor aqueous solubility or membrane permeability of the drug molecule. In the Biopharmaceutical Classification System (BCS), drugs with low aqueous solubility and high membrane permeability are categorized as Class II drugs. One of such drugs which fall under this category is telmisartan. One of the approaches which enhance the dissolution rate of poorly water-soluble drugs is "solid dispersions. Solid dispersions are a group of solid products consisting of a hydrophilic matrix and a hydrophobic drug [2]. As they increase the dissolution rate of drug at the absorption site, there is a gradual increase in the bioavailability too [3]. The solid dispersions were prepared using various techniques such as physical mixing, solvent evaporation, kneading, and fusion. Usage of certain superdisintegrants such as sodium starch glycolate, croscarmellose, and polyvinylpyrrolidine provides instantaneous degradation of the formulation. They are effective even at low concentrations. They were added to the formulation to promote the breakup of "slugs" into smaller fragments in an aqueous environment, thereby increasing the available surface area and promoting a more rapid release of the drug substance. They promote moisture penetration and dispersion [4]. Solid dispersions are investigated in many studies because they are highly versatile in their application. They can form the basis of products applied for various routes of administration and for various dosage forms, including the most popular dosage form, the tablet. Therefore, solid dispersion technologies are particularly promising for improving the oral absorption and bioavailability of BCS Class II drugs.
Natural superdisintegrants are preferable over synthetic forms, due to a wide range of availability, cost-effective, non-irritating, and non-toxic in nature. Various plant gums and mucilages also have superdisintegrant property [5]. These are mainly useful in improving the water solubility of BCS Class II drugs. These are the drugs with low solubility and high permeability properties. Past studies revealed the applications of components from plants like Ocimum tenuiflorum, Plantago ovata mucilage, Aloe vera mucilage, mucilage of Hibiscus rosa sinensis, and Lotus bean gum as superdisintegrants in formulating several BCS class II drugs.

In the present study, an attempt was made to extract starch from Entada scandens seed powder and to use it as superdisintegrant for preparing solid dispersions. The seed starch obtained from $E$. scandens possesses superdisintegrant property. E. scandens belonging to the family Fabaceae grows in evergreen tropical climates of India, Africa, and Australia. Fruits grow up to 6-m length with many seeds. Their seeds have a thick and durable seed coat which is brownish in color, which allows them to survive lengthy periods of immersion in seawater. It contains a fleshy white cotyledon [6]. They are rich in starch contents.

Telmisartan which is an antihypertensive agent is selected as the drug of choice for the present study. It belongs to the angiotensin receptor blocker class which mainly acts by inhibiting the angiotensin-II receptor. This prevents vasoconstriction and thereby reduces blood pressure. The absolute bioavailability of telmisartan depends on dosage. The drug is highly bound to plasma proteins (>99.5\%). It has an approximate terminal elimination half-life of $24 \mathrm{~h}$ [7]. Based on pharmacokinetic and pharmacodynamic parameters, telmisartan is selected as the drug of choice for the present study. 
The main aim of the current study was to extract starch from E. scandens seed powder and to formulate solid dispersions using it. Telmisartan solid dispersions were formulated with different concentrations of superdisintegrants using fusion technique. A novel formulation was prepared using the best formulation with maximum drug release and the starch extracted which could possibly prove the additive effect.

\section{MATERIALS AND METHODS}

\section{Materials}

Telmisartan was a gift sample from Mylan Laboratories Ltd. (Hyderabad, India). Sodium hydroxide was procured from S.D Fine Chem. Ltd. (Mumbai, India). Potassium dihydrogen orthophosphate and sodium lauryl sulfate were procured from Qualigens Fine Chemicals (Mumbai, India). Poloxamer-188 was a gift sample from M/s Pellets Pharma Ltd. (Hyderabad, India), and E. scandens seeds were procured from the local market (Kurnool, Andhra Pradesh, India)

\section{Extraction of starch from $E$. scandens seeds}

E. scandens seed starch was isolated using aqueous and alkali extraction methods [8]. $5 \mathrm{~g}$ E. scandens seed flour was added into $100 \mathrm{ml}$ distilled water and $0.1 \%, 0.25 \%$, and $0.5 \%$ sodium hydroxide solutions separately, soaked ( $6 \mathrm{~h}$ and $8 \mathrm{~h}$ ) at room temperature, and then stirred constantly. The slurry was filtered through 212 mesh stainless sieve and remaining sediment was washed with distilled water for 3 times. The filtrates were combined and precipitated overnight at $4^{\circ} \mathrm{C}$. The supernatant was discarded, and the crude starch was cleaned with distilled water. This step was repeated 3 times, and starch cake was dried at $40^{\circ} \mathrm{C}$ for $24 \mathrm{~h}$ in oven dryer. The starch was ground with a mortar and pestle. The starches were packed in a plastic bag and kept at room temperature until further use.

Phytochemical tests for E. scandens seed powder and extracted starches

The raw E. scandens seed powder and starches extracted were subjected to various phytochemical tests for the identification of carbohydrates, proteins, alkaloids, glycosides, steroids, flavonoids, and saponins [9]. These results are indicated in Table 1.

Evaluation of physicochemical properties of E. scandens seed powder and extracted starches

Various physicochemical properties were evaluated using suitable methods [9]. The results are indicated in Table 2.

Table 1: Phytochemical screening of Entada scandens seed powder and starches

\begin{tabular}{llllll}
\hline Test & ESSP & ESS1 & ESS2 & ESS3 & ESS4 \\
\hline Carbohydrates & + & + & + & + & + \\
Polysaccharides & + & + & + & + & + \\
Proteins & + & - & - & - & - \\
Alkaloids & + & - & - & - & - \\
Glycosides & - & - & - & - & - \\
Steroids & + & - & - & - & - \\
Flavonoids & + & - & - & - & - \\
Saponins & + & - & - & - & - \\
\hline
\end{tabular}

+ indicates present; - indicates absent

\section{Gelatinization temperature}

Samples of starch powder were moistened with water and loaded into capillary tube by means of intrusion. The temperature of gelling and the time from swelling to full gelatinization were measured with a melting point apparatus.

\section{Determination of $\mathrm{pH}$}

The $\mathrm{pH}$ values of $1 \%$ solutions were measured using a digital $\mathrm{pH}$ meter.

\section{Viscosity}

Flow property of a simple liquid is expressed in terms of viscosity. Viscosity is an index of resistance of a liquid to flow. The higher the viscosity of a liquid, the greater is the resistance to flow. The viscosity was measured using a Brookfield Viscometer.

\section{Swelling index}

About $1 \mathrm{~g}$ of powdered mucilage was treated with $25 \mathrm{ml}$ of water in a graduated cylinder shaken for every $10 \mathrm{~min}$ for $1 \mathrm{~h}$ and allowed to stand as specified.

$$
\text { Swelling Index }=\frac{\text { Weight of wet mass }}{\text { Weight of dry powder }} * 100
$$

\section{Water absorption index}

$1 \mathrm{~g}$ of sample was suspended in $10 \mathrm{ml}$ of distilled water at $30^{\circ} \mathrm{C}$ in a centrifuge tube, stirred for $30 \mathrm{~min}$ intermittently, and then centrifuged at $3000 \mathrm{rpm}$ for $10 \mathrm{~min}$. The supernatant was decanted, and the weight of the gel formed was recorded. The water absorption index was then calculated as gel weight per gram dry sample.

$$
\text { Water absorption index }=\frac{\text { Bound water }(\mathrm{g})}{\text { Weight of sample }(\mathrm{g})} * 100
$$

\section{Total microbial load of isolated E. scandens starch}

The total microbial load is an important parameter which decides the suitability of a substance for use as an excipient in pharmaceutical dosage form. The starch powders were subjected to dry heat sterilization at $180^{\circ} \mathrm{C}$ for $30 \mathrm{~min}$. Then, the starches were inoculated on medium and incubated for $24 \mathrm{~h}$. Then, the colonies were counted using a microbial colony counter

\section{Preparation of telmisartan solid dispersions by fusion method} (TP1 to TP7)

Poloxamer-188 was placed in China dish and heated at $40^{\circ} \mathrm{C}$ until it gets melted and then telmisartan was added to it. After vigorous stirring in normal temperature, the mixture was solidified. The solid mass was crushed, pulverized, and sieved. The granules obtained were stored in desiccators for further studies. Different formulations with various drug-to-polymer ratios are placed in Table 3.

Preparation of telmisartan solid dispersions by fusion method (TPS1 to TPS7)

After performing dissolution studies for the formulations TP1 to TP7, the formulation showing best dissolution profile was selected. To that

Table 2: Physicochemical properties of Entada scandens seed powder and extracted starches

\begin{tabular}{lllll}
\hline Properties & ESSP & ESS1 & ESS2 & ESS4 \\
\hline Gelatinization temperature $\left({ }^{\circ} \mathrm{C}\right)$ & $118-121$ & $120-123$ & $122-126$ & $124-127$ \\
pH & 6.32 & 6.50 & 6.62 & 6.78 \\
Viscosity (cps) & 1.856 & 2.089 & 2.166 & 2.265 \\
Swelling index & 58 & 63 & 74 & 76 \\
Water absorption index & Less & More & More & More \\
Total microbial load & Absent & Absent & Absent & Absent \\
\hline
\end{tabular}


Table 3: Composition of telmisartan solid dispersions prepared by fusion method using poloxamer-188

\begin{tabular}{lll}
\hline Formulation & Composition & Drug: polymer ratio (telmisartan: poloxamer-188) \\
\hline TP1 & Telmisartan: Poloxamer-188 & $1: 0.5$ \\
TP2 & Telmisartan: Poloxamer-188 & $1: 1.0$ \\
TP3 & Telmisartan: Poloxamer-188 & $1: 1.5$ \\
TP4 & Telmisartan: Poloxamer-188 & $1: 2.0$ \\
TP5 & Telmisartan: Poloxamer-188 & $1: 2.5$ \\
TP6 & Telmisartan: Poloxamer-188 & $1: 3.0$ \\
TP7 & Telmisartan: Poloxamer-188 & $1: 4.0$ \\
\hline
\end{tabular}

Table 4: Composition of telmisartan solid dispersions prepared by fusion method using TP7 and Entada scandens seed starch (ESS4)

\begin{tabular}{ll}
\hline Formulation & Percentage of ESS4 in formulation (\%) \\
\hline TPS1 & 2.0 \\
TPS2 & 2.5 \\
TPS3 & 5.0 \\
TPS4 & 7.5 \\
TPS5 & 10.0 \\
TPS6 & 12.5 \\
TPS7 & 15.0 \\
\hline
\end{tabular}

formulation, E. scandens seed starch (ESS4) was added in increasing concentrations, i.e., 2.0, 2.5, 5.0, 7.5, 10.0, and 12.5. The optimized formulation was kept constant in all these formulations. ESS4 was placed in China dish and heated at $40^{\circ} \mathrm{C}$ until it gets melted and then optimized telmisartan solid dispersion was added to it. After vigorous stirring in normal temperature, the mixture was solidified. The solid mass was crushed, pulverized, and sieved. The granules obtained were stored in desiccators for further studies. Different formulations with various solid dispersion-to-starch percentage ratio are placed in Table 4.

\section{Evaluation of pre-formulation parameters}

The prepared solid dispersions were evaluated for various preformulation parameters such as angle of repose, Carr's index, and Hausner's ratio [10]. These results are given in Table 5.

\section{Angle of repose}

The powder flow properties were determined to know the good or bad material flow by conducting this process in which powder was allowed to flow through a funnel to form a heap. Below the funnel tip, a graph paper was placed and the radius and heights of the heap were measured. The angle of repose was calculated by using the formula:

$$
\theta=\tan -1 \frac{h}{r}
$$

\section{Carr's index}

A simple test was used to evaluate the flowability of a powder by comparing the poured density and the tapped density of a powder and the rate at which it is packed down.

$$
\text { Carr'sindex }=\frac{\text { TappedDensity }- \text { PouredDensity }}{\text { TappedDensity }} \times 100
$$

\section{Hausner's ratio}

Hausner's ratio is an indirect index of the ease of powder flow. It is calculated by the following formula:

$$
\text { Hausner's ratio }=\frac{\text { TappedDensity }}{\text { Bulk Density }}
$$

Lower Hausner's ratio $(<1.25)$ indicates better flow properties than higher ones (>1.25).

\section{Drug content uniformity for solid dispersions}

Solid dispersions of telmisartan were taken and transferred into a $100 \mathrm{ml}$ volumetric flask, and $40 \mathrm{ml}$ of methanol was added to it. It was shaken occasionally for about $30 \mathrm{~min}$, and then, it was filtered using Whatman filter paper. From filtrate, $10 \mathrm{ml}$ was taken into and transferred to a $100 \mathrm{ml}$ volumetric flask, and the volume was made up to $100 \mathrm{ml}$ by adding $7.5 \mathrm{pH}$ phosphate buffer with $0.5 \%$ sodium lauryl sulfate. About $10 \mathrm{ml}$ of the solution from the volumetric flask was taken and centrifuged. Then, the filtrate was subsequently diluted and the absorbance was measured at $295 \mathrm{~nm}$. This test was repeated 6 times $(\mathrm{N}=6)$ for each formulation.

\section{In vitro dissolution studies of telmisartan solid dispersions}

Dissolution studies for each formulation were performed in a calibrated 8 station dissolution test apparatus (LABINDIA DS8000) equipped with paddles (USP apparatus II method) employing $900 \mathrm{ml}$ of phosphate buffer $\mathrm{pH} 7.5$ with sodium lauryl sulfate as a dissolution medium. The paddles were operated at $50 \mathrm{rpm}$, and the temperature was maintained at $37 \pm 1^{\circ} \mathrm{C}$ throughout the experiment. The samples were withdrawn at 5, 10, 15, 20,30,45, 60, 75, and $90 \mathrm{~min}$ and replaced with equal volume of same dissolution medium to maintain the constant volume throughout the experiment. Samples withdrawn at various time intervals were suitably diluted with the same dissolution medium, and the amount of the drug dissolved was estimated by LabIndia double beam U.V spectrophotometer (UV $3000+$ ) at $295 \mathrm{~nm}$. The dissolution studies on each formulation were conducted in triplicate. The dissolution profiles for all formulations are given in Tables 6 and 7.

\section{Statistical analysis}

The results obtained were statistically evaluated. As the procedures performed and the results obtained were in triplicate, the mean along with their standard deviations was calculated for drug content, and standard error of mean was calculated for drug dissolution profiles.

\section{Characterization studies}

Based on the dissolution studies, the optimized formulations were selected, and Fourier transfer infrared (FTIR) and differential scanning calorimetry (DSC) studies were performed to observe the drugpolymer interactions. X-ray diffraction (XRD) studies were performed to detect the nature of formulations. Scanning electron microscopy (SEM) analysis was performed on ESS4, telmisartan, poloxamer-188, and optimized solid dispersions TP7 and TPS7 to know surface characteristics. The results are shown in Figs. 1-4.

\section{RESULTS AND DISCUSSION}

Extraction of starch from $E$. scandens seeds

The starches extracted from E. scandens seeds were crisp slightly granular, free-flowing, and stable in nature. The starch extracted using water is given as ESS1. Similarly, the starch extracted using $0.1 \%$, $0.25 \%$, and $0.5 \%$ of sodium hydroxide was labeled as ESS2, ESS3, and ESS4, respectively.

Phytochemical screening of E. scandens seed flour and starch extracts

The raw E. scandens powder and starches extracted were screened for the presence of various phytochemical constituents. Seed powder showed the presence of all components except glycosides. The starches extracted showed only the presence of polysaccharides which shows that they does not contain any other phytochemicals that interfere with the results. The results are indicated in Table 1. 
Table 5: Pre-formulation parameters of telmisartan solid dispersions prepared by fusion method

\begin{tabular}{|c|c|c|c|c|c|}
\hline S. No & Formulation & Angle of repose $\left(^{\circ}\right)$ & Carr's index (\%) & Hausner's ratio & Drug content (mean $\pm S D$ ) \\
\hline 1 & TD & 33 & 20 & 1.22 & - \\
\hline 2 & TP1 & 26 & 18 & 1.19 & $16.85 \pm 0.45$ \\
\hline 3 & TP2 & 25 & 17 & 1.18 & $17.22 \pm 0.54$ \\
\hline 4 & TP3 & 23 & 17 & 1.18 & $17.85 \pm 0.41$ \\
\hline 5 & TP4 & 23 & 15 & 1.17 & $18.12 \pm 0.77$ \\
\hline 6 & TP5 & 22 & 13 & 1.16 & $17.54 \pm 0.81$ \\
\hline 7 & TP6 & 22 & 14 & 1.15 & $17.87 \pm 0.59$ \\
\hline 9 & TPS1 & 27 & 17 & 1.19 & $17.21 \pm 0.87$ \\
\hline 10 & TPS2 & 26 & 16 & 1.18 & $17.89 \pm 0.41$ \\
\hline 11 & TPS3 & 25 & 16 & 1.17 & $17.11 \pm 0.13$ \\
\hline 12 & TPS4 & 25 & 15 & 1.17 & $18.04 \pm 0.81$ \\
\hline 13 & TPS5 & 22 & 13 & 1.16 & $18.45 \pm 0.66$ \\
\hline 14 & TPS6 & 21 & 12 & 1.15 & $18.55 \pm 0.25$ \\
\hline 15 & TPS7 & 20 & 12 & 1.14 & $18.97 \pm 0.77$ \\
\hline
\end{tabular}

$\mathrm{n}=6$, SD: Standard deviation

Table 6: Dissolution profiles of telmisartan solid dispersions prepared by fusion method (TP1-TP7)

\begin{tabular}{|c|c|c|c|c|c|c|c|c|}
\hline \multirow[t]{2}{*}{ Time (min) } & \multicolumn{8}{|c|}{ Cumulative $\%$ drug released (mean \pm SEM) } \\
\hline & TD & TP1 & TP2 & TP3 & TP4 & TP5 & TP6 & TP7 \\
\hline 5 & $26.20 \pm 1.12$ & $27.44 \pm 1.25$ & $30.08 \pm 0.43$ & $35.44 \pm 0.57$ & $43.34 \pm 1.95$ & $46.84 \pm 0.43$ & $51.48 \pm 1.27$ & $58.86 \pm 0.64$ \\
\hline 10 & $30.90 \pm 0.89$ & $31.79 \pm 0.47$ & $34.63 \pm 1.22$ & $39.22 \pm 1.83$ & $46.07 \pm 0.41$ & $49.55 \pm 1.66$ & $54.29 \pm 0.53$ & $88.87 \pm 1.52$ \\
\hline 15 & $34.74 \pm 1.51$ & $35.42 \pm 1.93$ & $39.60 \pm 0.86$ & $43.54 \pm 1.40$ & $49.78 \pm 1.87$ & $52.98 \pm 1.78$ & $58.51 \pm 1.82$ & $96.50 \pm 0.84$ \\
\hline 20 & $36.68 \pm 0.64$ & $39.62 \pm 0.55$ & $42.99 \pm 1.10$ & $46.48 \pm 0.07$ & $52.58 \pm 0.62$ & $56.01 \pm 0.91$ & $62.43 \pm 2.31$ & $96.17 \pm 2.14$ \\
\hline 30 & $38.82 \pm 1.22$ & $42.96 \pm 1.82$ & $46.87 \pm 0.46$ & $49.68 \pm 2.82$ & $55.33 \pm 2.44$ & $59.51 \pm 2.25$ & $65.04 \pm 0.95$ & $96.37 \pm 1.49$ \\
\hline 60 & $40.65 \pm 1.31$ & $48.93 \pm 0.61$ & $56.22 \pm 0.29$ & $58.82 \pm 1.75$ & $62.12 \pm 1.54$ & $65.98 \pm 0.63$ & $71.45 \pm 0.88$ & $97.12 \pm 0.1 .75$ \\
\hline 75 & $41.87 \pm 1.56$ & $52.51 \pm 0.39$ & $61.57 \pm 1.83$ & $62.77 \pm 1.11$ & $65.31 \pm 1.43$ & $68.41 \pm 1.49$ & $75.01 \pm 1.57$ & $98.09 \pm 0.68$ \\
\hline 90 & $42.68 \pm 0.91$ & $55.57 \pm 1.22$ & $65.62 \pm 0.87$ & $66.34 \pm 0.67$ & $68.94 \pm 1.27$ & $71.88 \pm 1.19$ & $79.17 \pm 1.61$ & $98.14 \pm 1.43$ \\
\hline
\end{tabular}

$\mathrm{n}=3$, SEM: Standard error of mean

Table 7: Dissolution profiles of telmisartan solid dispersions prepared by fusion method (TPS1-TPS7)

\begin{tabular}{|c|c|c|c|c|c|c|c|c|}
\hline \multirow[t]{2}{*}{ Time (min) } & \multicolumn{8}{|c|}{ Cumulative $\%$ drug released (mean \pm SEM) } \\
\hline & TP7 & TPS1 & TPS2 & TPS3 & TPS4 & TPS5 & TPS6 & TPS7 \\
\hline 5 & $58.86 \pm 0.64$ & $66.15 \pm 2.24$ & $66.46 \pm 1.65$ & $68.2 \pm 1.22$ & $70.49 \pm 1.58$ & $74.86 \pm 1.08$ & $80.13 \pm 0.89$ & $84.52 \pm 1.07$ \\
\hline 10 & $88.87 \pm 1.52$ & $87.34 \pm 1.13$ & $88.39 \pm 1.89$ & $89.42 \pm 1.35$ & $90.13 \pm 1.05$ & $94.16 \pm 1.55$ & $96.16 \pm 1.19$ & $97.76 \pm 1.83$ \\
\hline 15 & $96.50 \pm 0.84$ & $98.04 \pm 1.65$ & $98.12 \pm 1.22$ & $98.24 \pm 0.84$ & $98.29 \pm 1.69$ & $98.36 \pm 1.94$ & $98.41 \pm 1.64$ & $98.98 \pm 0.99$ \\
\hline 20 & $96.17 \pm 2.14$ & $98.14 \pm 1.98$ & $98.22 \pm 1.05$ & $98.28 \pm 0.93$ & $98.34 \pm 2.05$ & $98.41 \pm 2.44$ & $98.52 \pm 1.83$ & $99.01 \pm 1.55$ \\
\hline 30 & $96.37 \pm 1.49$ & $98.21 \pm 1.05$ & $98.34 \pm 2.06$ & $98.39 \pm 1.54$ & $98.41 \pm 0.76$ & $98.45 \pm 2.31$ & $98.60 \pm 1.94$ & $99.12 \pm 0.72$ \\
\hline 45 & $96.76 \pm 2.41$ & $98.24 \pm 0.99$ & $98.42 \pm 1.84$ & $98.46 \pm 1.62$ & $98.49 \pm 1.39$ & $98.54 \pm 1.65$ & $98.74 \pm 2.16$ & $99.24 \pm 1.85$ \\
\hline 60 & $97.12 \pm 0.1 .75$ & $98.32 \pm 0.87$ & $98.46 \pm 0.91$ & $98.52 \pm 2.46$ & $98.56 \pm 1.60$ & $98.62 \pm 1.89$ & $98.81 \pm 2.58$ & $99.31 \pm 2.23$ \\
\hline 75 & $98.09 \pm 0.68$ & $98.35 \pm 1.24$ & $98.51 \pm 1.32$ & $98.59 \pm 2.11$ & $98.61 \pm 0.88$ & $98.68 \pm 0.84$ & $98.86 \pm 1.07$ & $99.40 \pm 1.94$ \\
\hline 90 & $98.14 \pm 1.43$ & $98.44 \pm 1.85$ & $98.56 \pm 1.77$ & $98.63 \pm 1.47$ & $98.68 \pm 2.65$ & $98.74 \pm 1.08$ & $98.92 \pm 1.66$ & $99.44 \pm 1.84$ \\
\hline
\end{tabular}

$\mathrm{n}=3$, SEM: Standard error of mean

Physicochemical parameters of $E$. scandens seed flour and starch extracts

Various physicochemical parameters were evaluated for the extracted starches, and the results are indicated in Table 2. The ESS4 starch was considered as the best suitable for formulations because it showed a pH nearer to body fluids, better viscosity, moderate swelling index, and more water absorption index. This gives the functional property of starch as polymer [11].

Preparation of telmisartan solid dispersions by fusion method Solid dispersions, TP1-TP7, were prepared by fusion method using Poloxamer-188 as carrier in different ratios keeping telmisartan constant. TD indicates telmisartan pure drug. Different formulations with a various drug to polymer ratios are placed in Table 3.

Similarly, solid dispersions, TPS1-TPS7, were prepared by fusion method using E. scandens seed starch (ESS4) as superdisintegrant in different ratios along with constant amounts of TP7. Different formulations with various drug to polymer ratios are placed in Table 4.

Evaluation of pre-formulation parameters

The pre-formulation parameter values obtained for various telmisartan solid dispersions were in the range of good flow characteristics and are given in the Table 4 . All the prepared granules were found to be stable and suitable for further studies.

In vitro dissolution studies of telmisartan solid dispersions Dissolution studies were carried on all solid dispersions using USP paddle method (apparatus II) with phosphate buffer pH 7.5 with $0.5 \%$ SLS as dissolution medium by maintaining the bath temperature at $37 \pm 1^{\circ} \mathrm{C}$ while the paddles were operated at $50 \mathrm{rpm}$. The dissolution profiles of all solid dispersions are given in Tables 6 and 7. The pure drug formulation, TM (pure drug) without any polymer, showed the lowest drug release in $90 \mathrm{~min}$, whereas the solid dispersion, TP7, prepared by fusion method exhibited maximum drug release. The solid dispersions prepared by fusion method has enhanced the dissolution 
rate which might be due to molecular inclusion of drug molecules into a long chain polymeric material containing hydrophobic and hydrophilic moieties [12,13]. It was also observed that as the concentration of poloxamer increased, the rate of dissolution of solid dispersions was also greatly increased. Past studies also revealed that fusion method is best to enhance the dissolution rate of poorly soluble drugs [14]. The dissolution profiles are indicated in Table 6.

Similarly, the dissolution studies were also carried out for the solid dispersions prepared using TP7 mixed with E. scandens seed starch (ESS4) to determine the carrier effect of starch. Invitro dissolution studies revealed that the formulation TPS7 prepared with 15\% of Entada scandens seed starch showed more drug release in less time. This indicates the superdisintegrant effect of starch. This strongly coincides with the recent studies with regard to the application of natural starches as superdisintegrants [15]. The dissolution profiles are indicated in Table 7.

\section{Characterization of telmisartan solid dispersions}

\section{FTIR spectral analysis}

The FTIR spectral investigations were carried out on pure drug of telmisartan, poloxamer-188, ESS4, and solid dispersions of telmisartan TP7 and TPS7. Telmisartan pure drug showed a broad peak at $3143.31 / \mathrm{cm}$ and sharp peaks at $2868.59 / \mathrm{cm}, 1100.64 / \mathrm{cm}$, and $862.64 / \mathrm{cm}$ indicating the presence of $\mathrm{NH} / \mathrm{OH}$ stretching, $\mathrm{CH}$ stretching, $\mathrm{C}-\mathrm{N} / \mathrm{C}-\mathrm{O}-\mathrm{C}$ cyclic ether stretching, and aromatic $\mathrm{CH}$ stretching. For poloxamer, a broad peak at $3499.85 / \mathrm{cm}$ and sharp peaks at $2880.40 / \mathrm{cm}$, $1103.79 / \mathrm{cmand} 842.24 / \mathrm{cm}$ indicate $\mathrm{NH} / \mathrm{OH}$ stretching, $\mathrm{CH}$ stretching, $\mathrm{C}-\mathrm{N} / \mathrm{C}-\mathrm{O}-\mathrm{C}$ cyclic ether stretching, and aromatic $\mathrm{CH}$ stretching. Starch extracted from E. scandens, ESS4, exhibited sharp peaks at 3049.42/ $\mathrm{cm}, 1645.74 / \mathrm{cm}, 1242.69 / \mathrm{cm}$, and $1014.79 / \mathrm{cm}$ indicating the presence of $\equiv \mathrm{C}-\mathrm{H}$ stretching, $\mathrm{C}=\mathrm{O}$ stretching, $\mathrm{C}=\mathrm{C}$ stretching, and $\equiv \mathrm{C}-\mathrm{O}$ - stretching. The solid dispersions TP7 and TPS7 showed a slight reduced intensity compared to that of drug and polymers, indicating that there were no drug-excipient interactions [16]. The detailed spectral elucidations were shown in Fig. 1.

\section{DSC thermogram analysis}

The DSC thermographic studies were carried out on pure drug of telmisartan, Poloxamer-188, ESS4, and solid dispersions of telmisartan TP7 and TPS7.

These studies exhibited broad endothermic peak at $278.96^{\circ}$ for telmisartan pure drug, a sharp endothermic peak at $59.51^{\circ} \mathrm{C}$ for Poloxamer-188, a broad endothermic peak at $311.1^{\circ} \mathrm{C}$ for ESS4, a broad endothermic peak at $268.43^{\circ} \mathrm{C}$, and a sharp endothermic peak at $59.48^{\circ} \mathrm{C}$ for $\mathrm{TP} 7$ formulation, indicating that there is a slight shift in temperature for drug. A sharp endothermic peak at $57.47^{\circ} \mathrm{C}$ and broad endothermic peaks at $283.24^{\circ} \mathrm{C}$ and $332.95^{\circ} \mathrm{C}$ for formulation TPS7 were observed, indicating that there is a slight shift in temperature for drug and starch. On the basis of this thermal behavior, drug and excipients used in this study were found to be compatible with each other. The detailed thermographs are shown in Fig. 2.

\section{SEM analysis}

SEM images were taken for pure drug telmisartan, poloxamer-188, ESS4, and solid dispersions TP7 and TPS7. Telmisartan pure drug exhibited crystalline form. Poloxamer-188 exhibited free-flowing large spherical forms. The ESS4 starch exhibited free-flowing spherical low dense form of starch grains without any mucilage/resinous coverage. The SEM image of TP7 solid dispersion clearly exhibited uniform dispersion of drug with poloxamer-188. The SEM image of TPS7 solid dispersion clearly exhibited uniform dispersion of drug with poloxamer-188 and starch grains. The obtained results could be attributed to the dispersion of drug in the molten mass of polymer [17]. The detailed SEM images are shown in Fig. 3.

\section{XRD analysis}

XRD diffraction studies were carried out for pure drug telmisartan, poloxamer-188, ESS4, and solid dispersions TP7 and TPS7.

The XRD of telmisartan showed its typical crystalline pattern. Poloxamer-188 showed crystalline nature. ESS4 showed amorphous nature. Formulation, TP7 which is a solid dispersion made of telmisartan and poloxamer-188, showed diffused peaks indicating the amorphous nature of the solid dispersions. Formulation, TPS7 which is a mixture of TP7 solid dispersions and $15 \%$ of ESS4 starch, showed diffused peaks indicating the amorphous nature of the solid dispersions. These results indicated that the drug was bound well with the polymers used which were indicated by the change in phase [18]. The detailed diffractograms are shown in Fig. 4. Several studies conducted in the past also revealed that solid dispersion forms enhanced the solubility of poorly watersoluble drugs [19].

\section{CONCLUSION}

The starch ESS4, extracted from E. scandens seeds by $0.5 \%$ sodium hydroxide, is found to be the best and is used as superdisintegrant for the preparation of solid dispersions. Telmisartan solid dispersions were prepared using various concentrations of ESS4 and Poloxamer-188 and were subjected to in vitro dissolution studies. From these studies, it was observed that the proportion of starch as superdisintegrant has

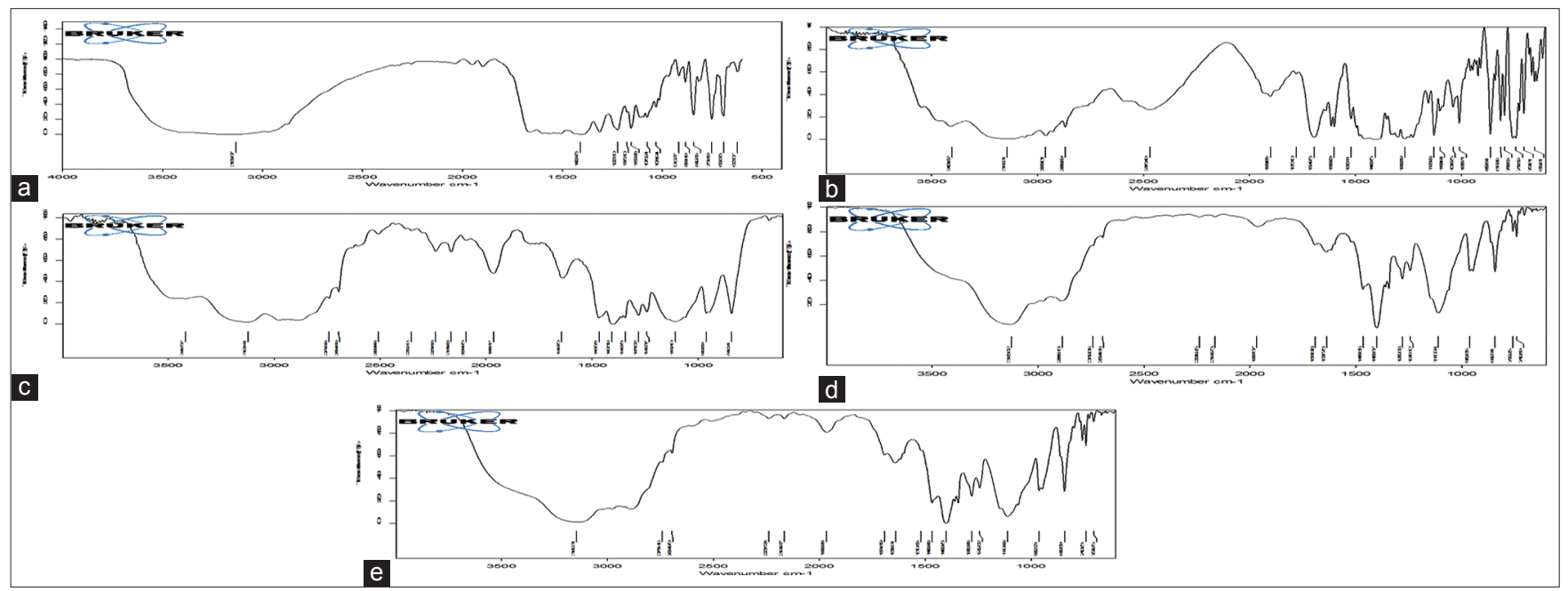

Fig. 1: Fourier transfer infrared spectra: (a) Telmisartan (b) Poloxamer-188 (c) ESS4 (d) TP7 (e) TPS7; ESS4: Entada scandens seed starch extracted by $0.5 \%$ sodium hydroxide; TP7: Solid dispersion of telmisartan+poloxamer-188 in 1:4 ratios; TPS7: Solid dispersion of TP7 with $15 \%$ ESS4 


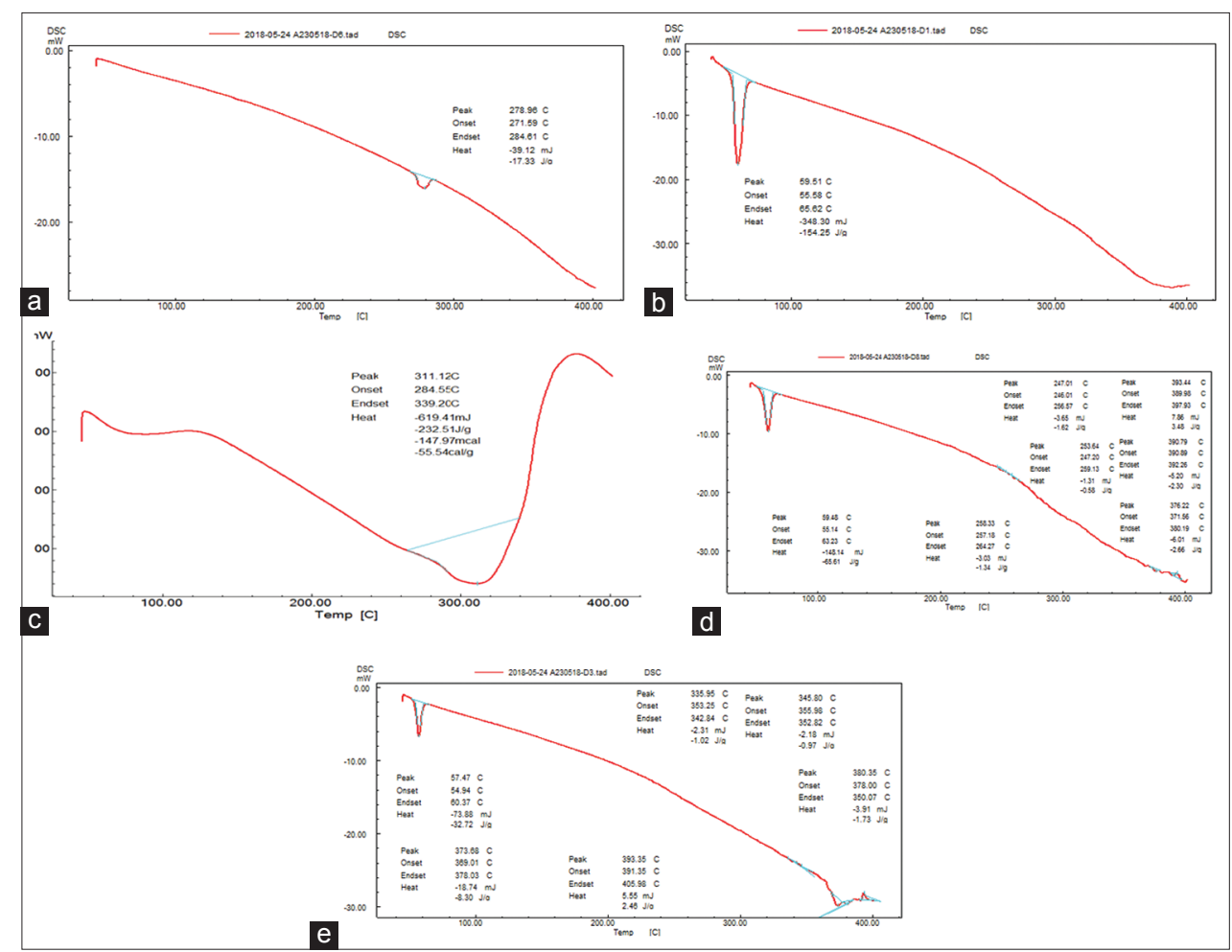

Fig. 2: Differential scanning calorimetry thermograms: (a) Telmisartan, (b) poloxamer-188, (c) ESS4, (d) TP7 (e) TPS7; ESS4: Entada scandens seed starch extracted by $0.5 \%$ sodium hydroxide; TP7: Solid dispersion of telmisartan+Poloxamer-188 in 1:4 ratios; TPS7: Solid dispersion of TP7 with $15 \%$ ESS4

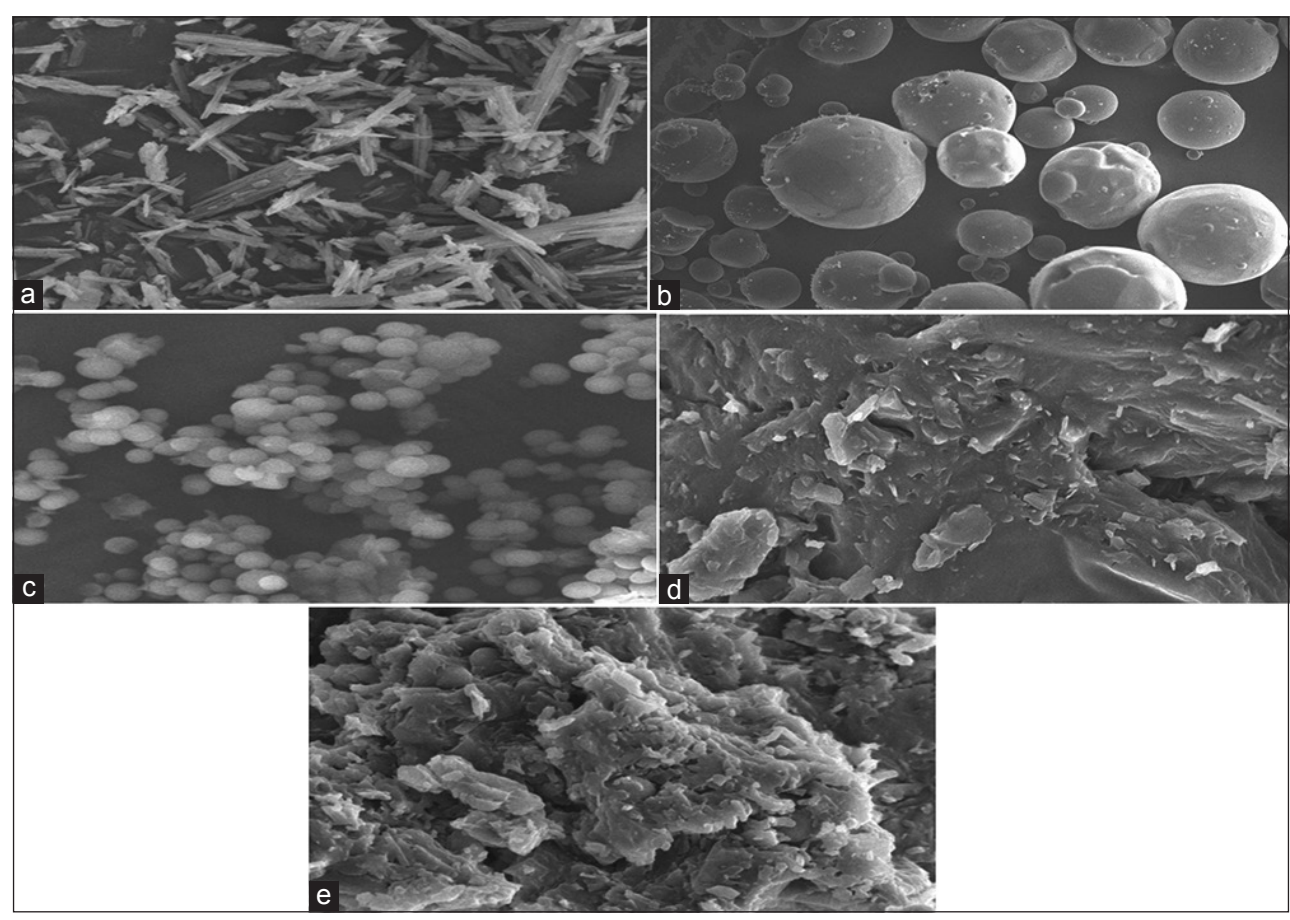

Fig. 3: Scanning electron microscopy images: (a) Telmisartan, (b) poloxamer-188, (c) ESS4, (d) TP7, (e) TPS7; ESS4: Entada scandens seed starch extracted by $0.5 \%$ sodium hydroxide; TP7: Solid dispersion of telmisartan+poloxamer-188 in 1:4 ratios; TPS7: Solid dispersion of TP7 with 15\% ESS4

influenced the dissolution parameters of various formulations. Higher dissolution rate was observed for formulation, TPS7 containing $15 \% \mathrm{w} / \mathrm{w}$ of ESS4 as superdisintegrant mixed with the solid dispersion TP7. The possible mechanism of superdisintegrant effect of these starches might be the rapid uptake of water, followed by swelling which causes the elevation of hydrostatic pressure that leads to faster dispersion. The optimized formulations were subjected to FTIR and DSC analysis to study the drugexcipient interactions, which revealed no such interactions. Similarly, XRD 


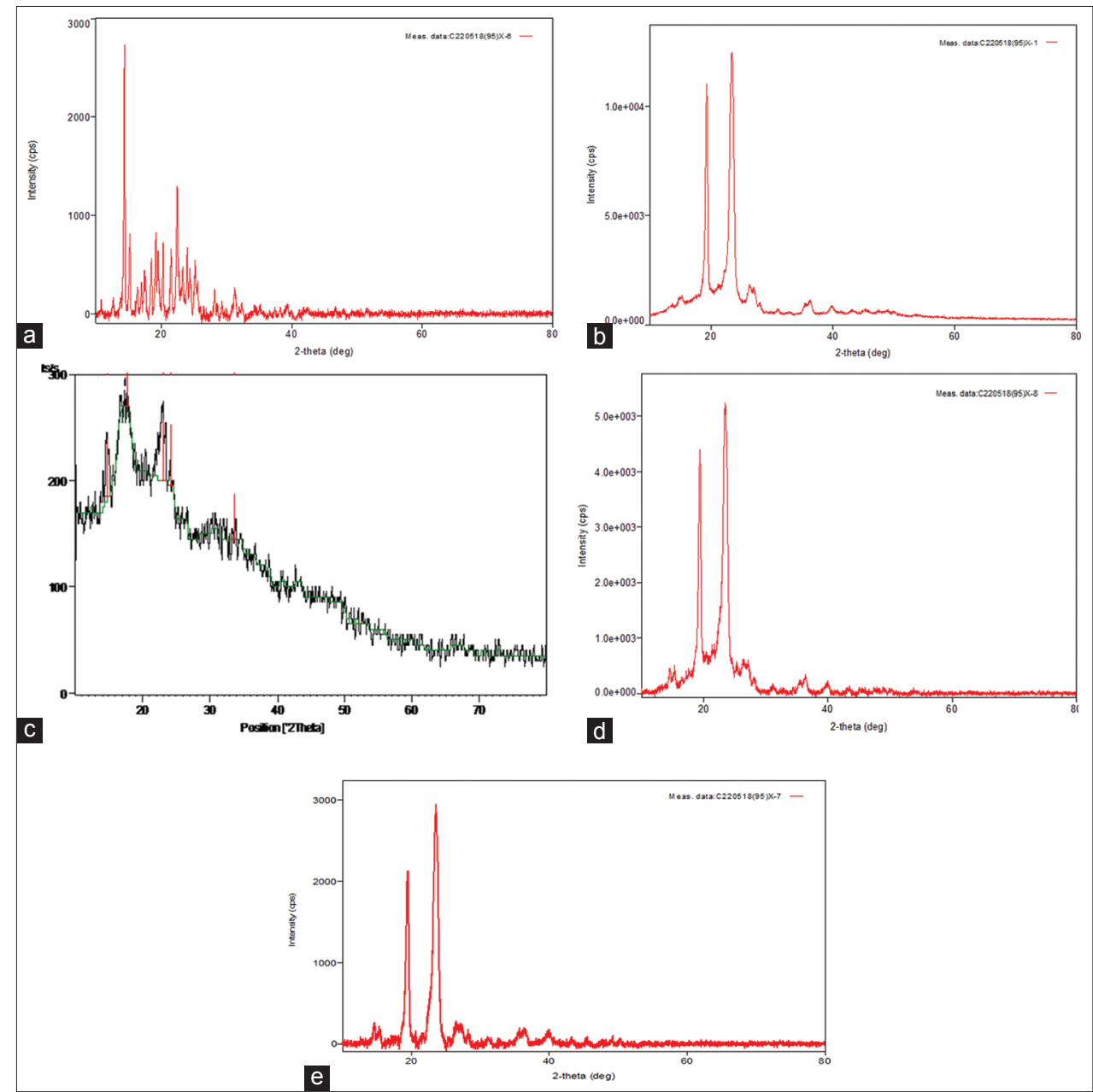

Fig. 4: X-ray diffraction: (a) Telmisartan (b) poloxamer-188 (c) ESS4, (d) TP7, (e) TPS7; ESS4: Entada scandens seed starch extracted by $0.5 \%$ sodium hydroxide; TP7: Solid dispersion of telmisartan+poloxamer-188 in 1:4 ratios; TPS7: Solid dispersion of TP7 with 15\% ESS4

studies were conducted to know the crystalline and amorphous nature of the samples. Based on the above studies, it may be concluded that the telmisartan solid dispersions prepared using $0.5 \%$ sodium hydroxide extracted E. scandens seed starch showed rapid drug release.

\section{ACKNOWLEDGMENTS}

The authors are thankful to the management of Chebrolu Hanumaiah Institute of Pharmaceutical Sciences for their sheer support throughout the work. The authors also express their thanks to M/s. Mylan Laboratories Ltd. (Hyderabad, India) for their generous gift sample of telmisartan and Dr. D. Hari Narayana, Nishka Labs, Hyderabad, for their extensive support in conducting DSC, SEM, and XRD studies.

\section{AUTHOR'S CONTRIBUTION}

Each author has contributed to the best of our knowledge for the research work carried out in this article.

\section{CONFLICTS OF INTEREST}

The authors declare no conflict of interest.

\section{REFERENCES}

1. Chiou WL, Riegelman S. Pharmaceutical applications of solid dispersion systems. J Pharm Sci 1971;60:1281-302.

2. Priya P, Rajendran NN, Lakshmi PK, Umadevi SK, Kaushalya J. A novel captopril hydrochlorthiazide solid dispersion. Int J Pharm Pharm Sci 2010;2:30-2.

3. Chauhan B, Shimpi S, Paradkar A. Preparation and evaluation of glibenclamide-polyglycolized glycerides solid dispersions with silicon dioxide by spray drying technique. Eur J Pharm Sci 2005;26:219-30.

4. Bhowmik D, Chiranjib B, Yadav J, Chandira RM, Sampath KP. Emerging trends of disintegrants used in formulation of solid dosage form. Schol Res Lib 2010;2:495-504.

5. Sunada H, Bi Y. Preparation, evaluation and optimization of rapidly disintegrating tablets. Powder Technol 2002;122:188-98.

6. Dey SK, Rahman M, Ahmed A, Khatun A, Siraj A. Phytochemical screening and pharmacological activities of Entada scandens seeds. Int J Appl Res Nat Prod 2013;6:34-9.

7. Benson SC, Pershad SH, Chittiboyina A, Desai P, Pravenec M, Wang J. Identification of telmisartan as a unique angiotensin II receptor antagonist with selective PPAR-modulating activity. Hypertension 2004;43:993-1002

8. Fateatun N, Jiaur RM, Sultan MM, Ahmed M. Physicochemical properties of flour and extraction of starch from jack fruit seed. Int $\mathrm{J}$ Nut Food Sci 2014;3:347-54

9. Menaka T, Nagaraja G, Yogesh DB, Sunil US, Prakash L. Physicochemical properties of flour and isolated starch from jack fruit seeds. Res J Pharm Sci 2011;01:14-8.

10. Okunlola A, Odeku OA. Comparative evaluation of starches obtained from Dioscorea species as intragranular tablet disintegrants. J Drug Del Sci Technol 2008;18:445-7.

11. Hetal P, Mukesh G. A review on development of multifunctional coprocessed excipient. J Crit Rev 2016;3:48-54.

12. Ghareeb MM, Abdulrasool AA, Hussein AA, Noordin MI. Kneading technique for preparation of binary solid dispersion of meloxicam with poloxamer 188. AAPS Pharm Sci Tech 2009;10:1206-15.

13. Quadir A, Kolter K. A comparative study of current superdisintegrants. Pharm Technol 2006;10:1-4.

14. Shashikumar Y, Veena M, Srinivas M. Solid dispersion technique to 
enhance the solubility and dissolution rate of aripiprazole by fusion method. Int J Pharm Pharm Sci 2016;8:187-92.

15. Vidyadhara S, Sasidhar RL, Lakshmi HD, Vijetha P, Vijetha K. Studies on jack fruit seed starch as a novel natural superdisintegrant for the design and evaluation of irbesartan fast dissolving tablets. Int Med Res 2017;6:280-91.

16. Gubbi S, Jarag R. Liquisolid technique for enhancement of dissolution properties of bromhexine hydrochloride. Res J Pharm Technol 2009;2:382-6.
17. Anshu S, Chandra PJ, Yuveraj ST. Prepartaion and characterization of solid dispersions of carvedilol with poloxamer-188. J Chil Chem Soc 2013;58:1553-7.

18. Vipan KK, Prabhakar KV. Preparation and characterization of metformin loaded stearic acid coupled F127 nanoparticles. Asian J Pharm Clin Res 2018;11:212-7.

19. Isha S, Shailendra B, Alpesh Y. Enhancement of solubility and dissolution of nebivolol by solid dispersion technique. Int J Pharm Pharm Sci 2014;6:566-71. 